\title{
Angle Robust Reflection/Transmission Plasmonic Filters Using Ultrathin Metal Patch Array
}

\author{
Chenying Yang, Weidong Shen, * Jing Zhou, Xu Fang, Ding Zhao, Xing Zhang, \\ Chengang Ji, Bo Fang, Yueguang Zhang, Xu Liu, and L. Jay Guo
}

Color filter, as a common optical element, can be generally grouped in two categories in the light of the filtering mechanism: chemical color filter and optical color filter. Traditional chemical color filters realize the color filtering feature through the wavelength-selective absorption of the specific functional groups of the pigments and dyes, which are not stable to a variety of processing chemicals, cannot suffer long-time strong illumination, and will cause a significant environmental burden simultaneously. On the other hand, the optical thin film filters by multilayer interference perform well with a high peak transmittance, a tailored bandwidth, and stable specifications. Though some researches have been carried out to lower the angular dependence of the thin film filters with metaldielectric-metal or similar structures, ${ }^{[1-3]}$ the transmission/ reflection spectra move inevitably with the incidence angle. Plasmonic filters, recently as a research hotspot of the optical filters, have attracted a wide range of interests both in academic and industrial communities for the unique and extraordinary optical properties as well as the extensive potential applications in the fields of display, detecting, printing, decoration, and so forth. ${ }^{[4-23]}$ In recent decades, plasmonic filters with various specific nanostructures have been investigated broadly and developed in different aspects, mostly based on the surface plasmon resonance within the metallic nanostructures. However, almost all the plasmonic filters presented previously were quite sensitive to the angle of incidence, which severely limits numerous applications under large angle of oblique incidence.

Dr. C. Yang, Dr. W. Shen, Dr. X. Fang,

Dr. D. Zhao, B. Fang, Dr. Y. Zhang, Prof. X. Liu

State key Laboratory of Modern

Optical Instrumentation

Department of Optical Engineering

Zhejiang University

Hangzhou 310027, China

E-mail: adongszju@hotmail.com

Dr. C. Yang, Dr. J. Zhou, C. Ji, Prof. L. J. Guo

Department of Electrical Engineering and Computer Science University of Michigan

Ann Arbor, Michigan 48109, USA

Dr. W. Shen

Haixi Collaborative Innovation Center for

New Display Devices and Systems Integration

Fuzhou University

Fuzhou 350002, China

$X$. Zhang

Science and Technology on Special System Simulation Laboratory

Beijing Simulation Center

Beijing 100854, China

DOI: 10.1002/adom.201600397
Though Yi-Kuei Ryan Wu proposed a novel scheme to achieve angle insensitive color filtering through the localized resonance in metallic nanoslits by light funneling, only transverse magnetic (TM) polarization of the incident light was considered because of its intrinsic 1D grating structure and cone of the incident light characterized by the azimuthal angle was also neglected, both of which limit its further application in many fields. ${ }^{21]}$ This year, Luc Duempelmann presented a plasmonic color filter based on periodic subwavelength silver nanowires, capable of changing the output color by simple rotation of a polarizer, and lower angular dependence can be observed in this scheme. ${ }^{[22]}$ But this plasmoic filter has a low transmission and the angle insensitivity for $\mathrm{p}$ and s polarizations could be further improved. And in our previous work, ${ }^{[23]}$ an omnidirectional reflective color filter based on metal-dielectric-metal (MDM) subwavelength grating structure was proposed, which can offer a high incident angular tolerance with the reflection dip wavelength invariable up to $60^{\circ}$. Nevertheless, the bandwidth of the filter changes gradually with the increasing incidence angle, which limits the potential applications in fields of sensing, detecting, and spectral analysis. Besides, the diffusion between the ultrathin metallic layer and the dielectric film is also a big challenge for a longtime stability.

Here, a new omnidirectional plasmonic filter based on ultrathin metal patch array structure is proposed, which can present the same perceived reflection/transmission color at unpolarized illumination for a broad range of incident angles. The reflection/transmission curves are coincident at different angles and the color difference characterized by CIE DE 2000 is inconceivably small, implying that no color variation can be observed for a large angle up to $60^{\circ}$. The plasmonic filter can cover the whole visible wavelength range by simply adjusting the dimension of the patch structure, which is of great benefit for the realization of the complex colorful pattern with convenient manufacturing process. The localized surface plasmonic resonance excited in the single metallic patch is responsible for the angle insensitive color filtering feature of this plasmonic filter. And because of this, patch array with as few as two periods was sufficient to demonstrate color filtering.

Figure 1a shows the schematic geometry of the angle robust reflection/transmission plasmonic filter, which is comprised of a single layer of ultrathin metal patch array structure. It is noted that such simple structure of ultrathin metal patch array has been used to realize coding metasurfaces to control the reflections and scattering of electromagnetic waves. ${ }^{[24]}$ Here, nanocuboid is chosen as the patch pattern in our study, and the patch pattern is not important due to the physical origin of its angle insensitive color filtering, which will be discussed in the 
(a) Incident
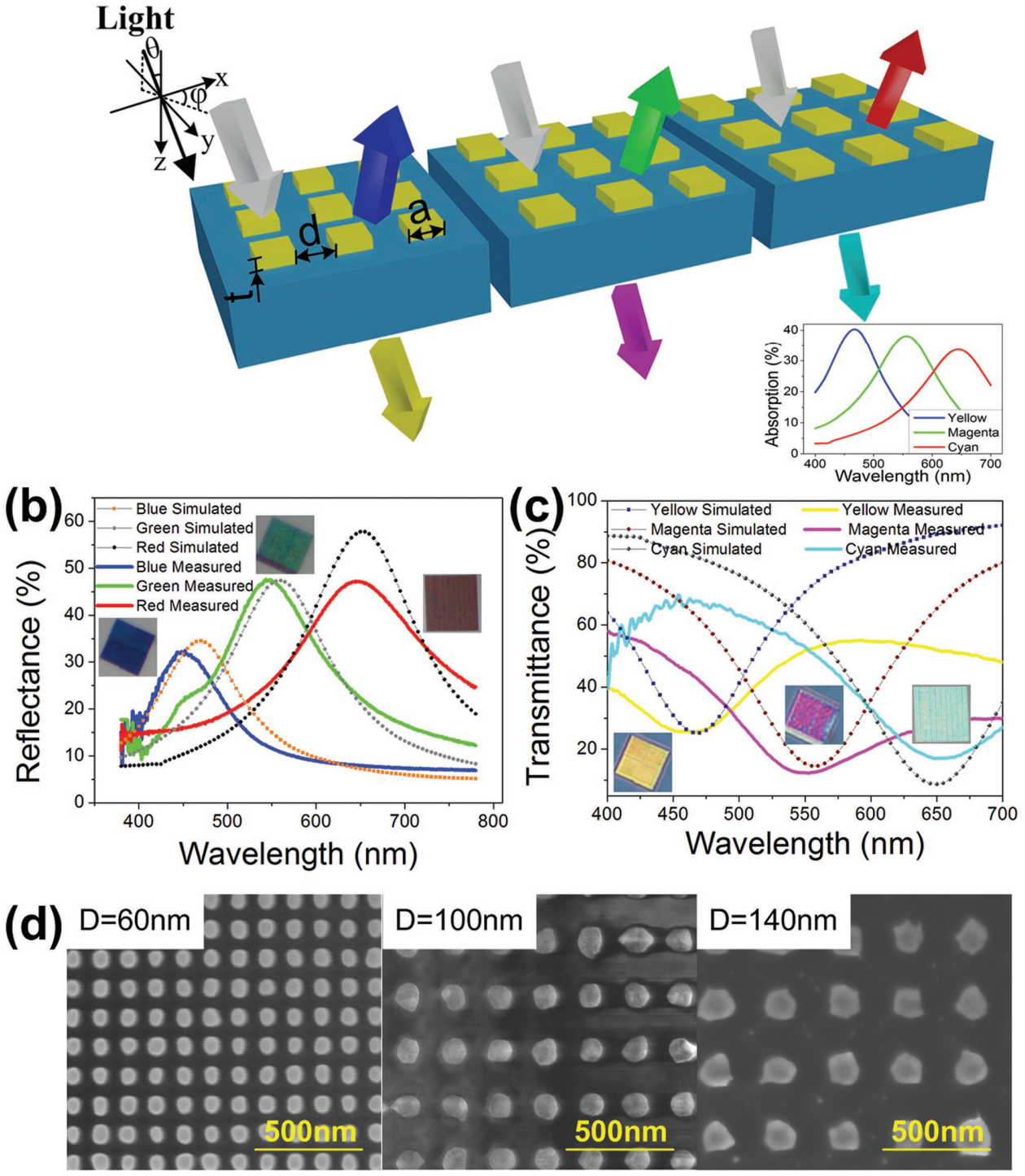

Figure 1. a) The schematic geometry of the angle robust reflection/transmission plasmonic filters using ultrathin metal patch array structure. The calculated absorption of the proposed filters is shown in the inset. b) The simulated and measured reflectance curves of the reflective RGB filters of nanodisks pattern at normal incidence with their corresponding photo images. c) The simulated and measured transmittance curves of the transmissive CMY filters of nanodisk pattern at normal incidence and their corresponding photo images. d) The top view SEM images of the reflective RGB/ transmissive CMY filters with specific patch sizes.

later section of this paper. The thickness of the patch is denoted by $t$, the patch size of the nanocuboid is denoted by $a$, and the interpatch distance between the adjacent nanocuboids is represented by $d$. The duty ratio of the patch is defined as $f=a /(a+d)$. The materials of incident medium and substrate are air and silica, whose refractive indices are $n_{0}=1.0$ and $n_{\mathrm{s}}=1.52$ respectively. Silver (Ag) is selected as the material of the patch, whose refractive index and extinction coefficient come from the data in the book ${ }^{[25]}$ (the data between the nodes derived from linear interpolation). A linearly polarized light is launched at an incident angle $\theta$ and an azimuthal angle $\varphi$ toward the front side of the color filter. With the patch thickness to be $20 \mathrm{~nm}$ and the duty ratio to be $50 \%$, reflective red, green and blue (RGB) colors can be achieved at the period of 70, 140, and $240 \mathrm{~nm}$, respectively. Correspondingly, the transmissive colors are cyan, magenta, and yellow (CMY). The geometry is designed with the help of particle swarm optimization and appropriate merit functions. ${ }^{[23,26,27]}$ A commercial software FDTD Solutions, which is based on finite-difference time-domain (FDTD) method, ${ }^{[28-30]}$ is adopted in our study to calculate the reflectance/transmittance of the filters as well as the electric field distribution of the structure. And the devices are fabricated by steps of sputtering deposition and focus ion beam (FIB) milling. The photos and scanning electron microscope (SEM) images of devices corresponding to RGB/CMY colors are displayed in Figure 1b-d. However, due to fabrication imperfection, the corners of the 
nanocuboids are rounded. Therefore, according to the FIB results shown in Figure 1d, the practical structural parameters can be read out for different colors and the simulated results of the nanodisk pattern with parameters are calculated as well, shown in Figure 1b,c. Obviously, the measured results agree well with the simulated results and the peak reflectance can reach $30 \%$ or more for reflective RGB filters, providing enough luminance in applications. On the other hand, the simulated transmittance of $80 \%$ or more for the high transmission region can be observed, which not only enhances luminance but also improves saturation. The difference of the simulated and measured transmittance for the high transmission region can be observed, which can be attributed to the oxidization of the material Ag. The transmittance of the micro-area device is measured by the self-made conjugate optical measurement system, while the reflectance is measured by a spectrophotometer (OLYMPUS USPM-RU). As well, the absorption of these color devices is calculated and shown in the inset of Figure 1a. Distinguished from the little absorption for the uniform Ag film of $20 \mathrm{~nm}$, the proposed devices present distinct absorption peaks for different colors corresponding to these reflection peaks and transmission dips, which could be attributed to the localized surface plasmon resonance excited within the structure.

With such simple structure of ultrathin metal patch array, the plasmonic filters can present good angle insensitivity feature. Figure 2 shows the transmittance of the CMY color filters at different incidence angles for average polarization. For cyan and magenta filters, the simulated results of the nanocuboid pattern in Figure $2 \mathrm{a}, \mathrm{b}$ indicate that the transmission spectrum almost remains the same for different incidence angles. When the size of the patch pattern increases to construct the cyan filter, the angle insensitivity property deteriorates gradually. But the cyan filter has a relatively high angular tolerance compared with those reported plasmonic filters. The simulated transmittances of the nanodisk pattern shown in Figure 2c match roughly with the measured transmittances shown in Figure $2 \mathrm{~d}$ and the difference of the transmittance values could be attributed to the deviation between the fabricated pattern and simulated pattern of ideal nanodisk pattern. Based on the transmission spectra, the transmitted colors are calculated by colorimetry theory as marked in a CIE 1931 chromatic diagram in the Supporting Information. These filters can have the identical color appearance at different angles. The color difference characterized by CIE DE2000 formula ${ }^{[31-33]}$ of these filters is limited within 2 up to $30^{\circ}$ or more (The calculated CIE DE 2000 values of these optimized filters can be seen in the Supporting Information), which is incredible for the so-far reported color filters. In comparison with the previous MDM filter, ${ }^{[23]}$ the filter here presents better angle insensitivity with the invariable bandwidth and less color difference. Because of the increased dimension, the color difference of the cyan filter deteriorates sharply, which can be suppressed through the smaller patch size by decreasing the thickness of the patch. It should be noted that the angular performance of the patch array structure with the pattern of nanocuboid is much better than that of the patch array structure with nanodisks, which can be verified in the Supporting Information.

To reveal the physical origin of the spectral filtering feature of the proposed omnidirectional color filter, we investigated the patch array with a lateral size of $70 \mathrm{~nm}$ and a thickness of
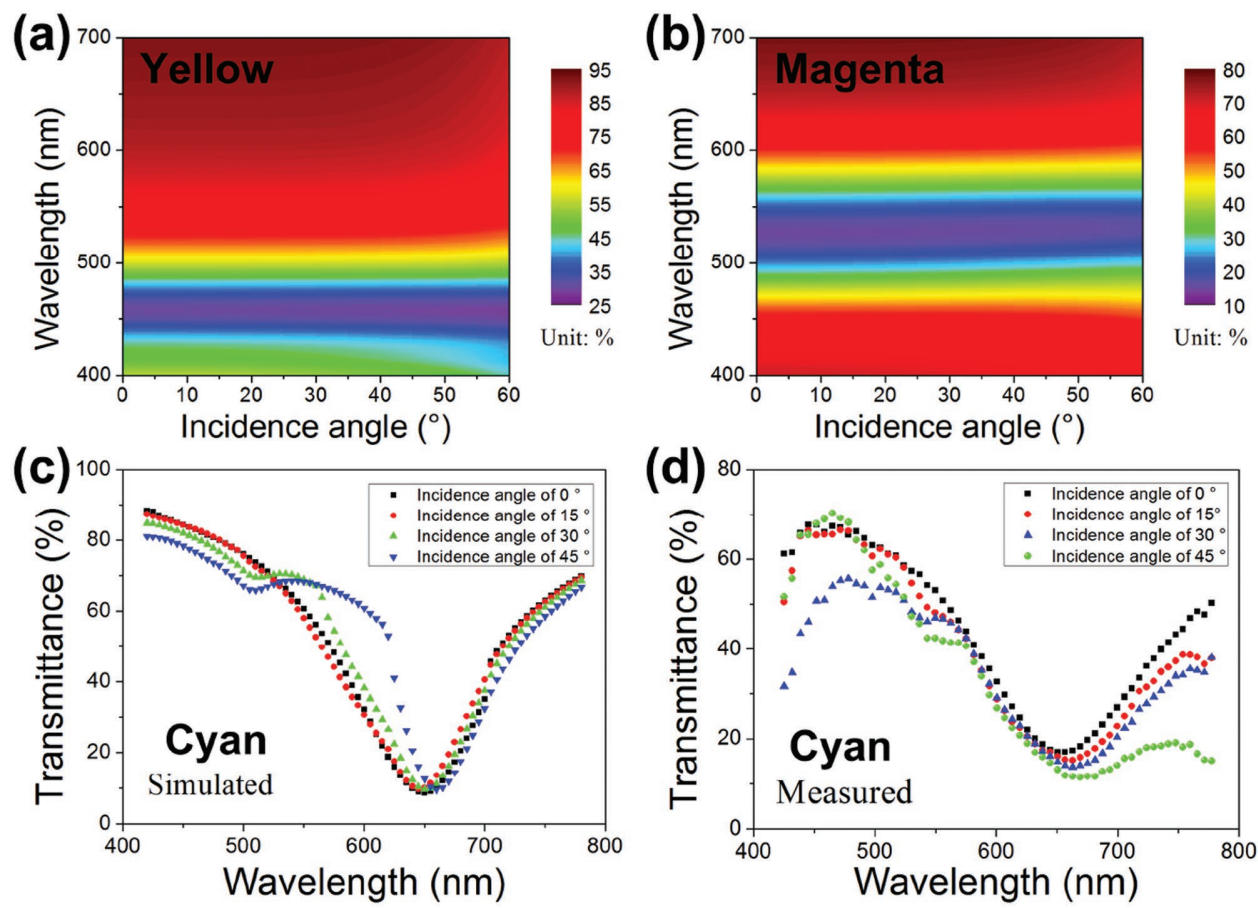

Figure 2. a) Simulated transmittance of the transmissive yellow filter of the nanocuboid pattern as a function of wavelength and the angle of incidence for average polarization. b) Simulated transmittance of the transmissive magenta filter of the nanocuboid pattern as a function of wavelength and the angle of incidence for average polarization. c) Simulated transmittance curves of the transmissive cyan filter of the nanodisk pattern at different incidence angles for average polarization. d) Measured transmittance curves of the transmissive cyan filter at different incidence angles for average polarization. 


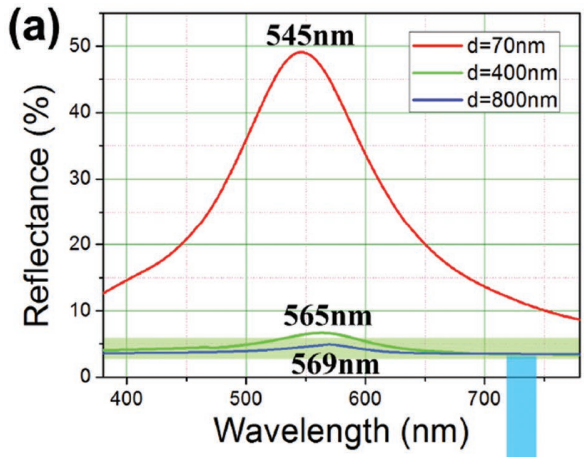

(b)
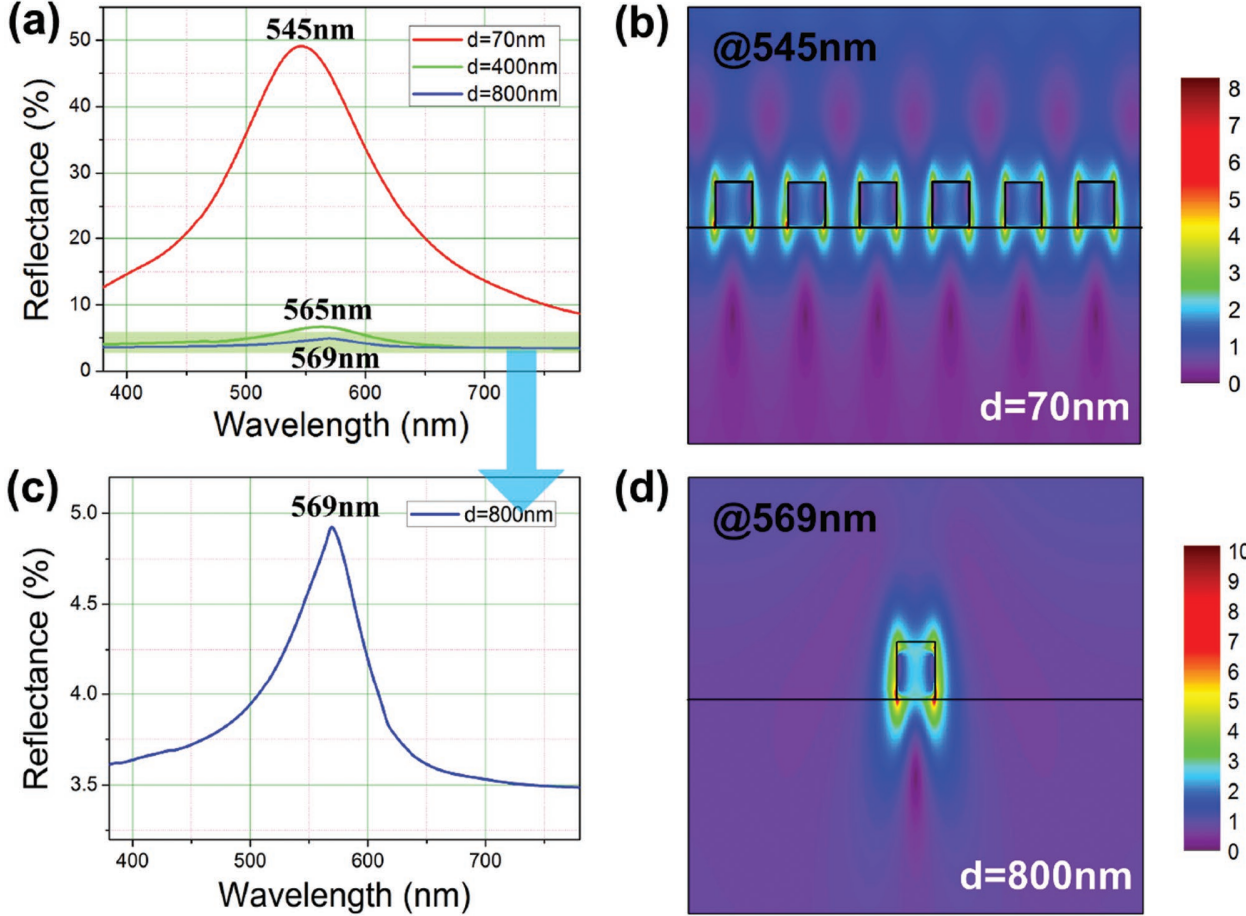

(d)

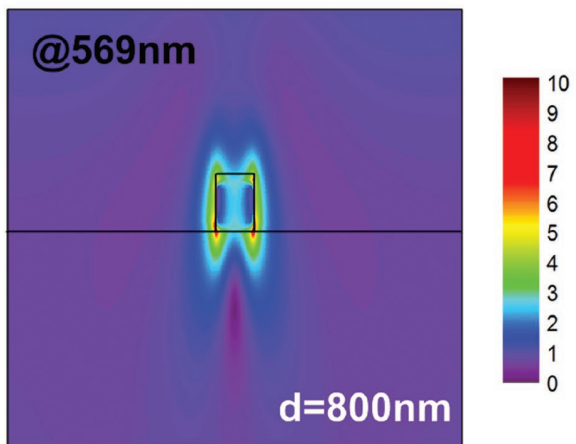

Figure 3. a) The reflectance curves of the structure for the reflective green filter with three different interpatch distances $d=70,400,800 \mathrm{~nm}$. b) The electric field distribution of the patch array structure with the inter-patch distance $d=70 \mathrm{~nm}$ for the peak reflectance wavelength $\lambda=545 \mathrm{~nm}$. c) The magnified reflectance curve of the patch array structure with the interpatch distance $=800 \mathrm{~nm}$. d) The electric field distribution of the patch array structure with the interpatch distance $d=800 \mathrm{~nm}$ for the peak reflectance wavelength $\lambda=569 \mathrm{~nm}$.

$20 \mathrm{~nm}$ at different interpatch distances $(70,400$, and $800 \mathrm{~nm})$. Figure 3a shows the reflectance curves with different interpatch distances, while the magnified reflectance curve with $d=800 \mathrm{~nm}$ is shown in Figure 3c. It is not difficult to find that the reflection peaks are located closely (@545, @565, @569 nm) for the cases of three interpatch distances, which means that the distances between the patches have almost no impact on the resonance wavelength but the intensity of the peak reflection. Since various colors can be produced by different periods, it is the size of the patch pattern that determines the center wavelength. It is noted that the optically thick metal films with perforated nanostructures can present EOT and behave as the transmissive RGB filters, attributted to the excitation of surface plasmon polaritons. For our proposed structure, the principle of the enhanced transmission, the modulated reflection as well as the particular absorption is totally different. Figure 3b shows the electric field profile of the patch array structure with interpatch distance $d=70 \mathrm{~nm}$ for peak reflectance wavelength $\lambda=545 \mathrm{~nm}$, while Figure $3 \mathrm{~d}$ shows the electric field of the patch array structure with interpatch distance $d=800 \mathrm{~nm}$ for peak reflectance wavelength $\lambda=569 \mathrm{~nm}$. It is apparent that dipole-like resonances are excited within the patch structure for two structures with different interpatch distances. For the patch array structure with the interpatch distance $d=800 \mathrm{~nm}$, which is far more than the original interpatch distance, the filtering feature of this case resulted from the effect of the single metallic nanopatch. As a matter of fact, the single metallic patch with such small thickness and tiny size can only support localized surface plasmon resonance. ${ }^{[34,35]}$ When the interpatch distance decreases to 70 $\mathrm{nm}$, the full width half maximum grows broad compared with that for large interpatch distance, which implies that a weak coupling between the patches is excited in the structure with the interpatch distance $d=70 \mathrm{~nm}$ or so, as shown in Figure $3 \mathrm{~b}$. On the other hand, the localized surface plasmon resonance is excited by the specific nanostructures of the surface with the appropriate polarization and frequency of the incident light and the resonance frequency is determined by the shape and dimension of the nanostructures, which is independent of the incident wave vector (i.e., the incidence angle). Therefore, the localized surface plasmon resonance excited by the structure accounts for the angle robust spectral filtering of this reflection/transmission plasmonic filter.

Based on the analysis of the physic origin for the angle insensitive color filtering, we further study the smallest pixel to realize the color filtering sufficiently. Figure 4 shows the SEM images and corresponding photo images with $2 \times 2$ array, $4 \times 4$ array, $6 \times 6$ array, $8 \times 8$ array for the transmissive yellow/reflective blue filter. The transmissive yellow/reflective blue colors are kept with the periods of the pixel decreased down to two periods. The color seems vague when the pixel constituted by $2 \times 2$ array, and it is because the pixel dimension $(\approx 200 \mathrm{~nm})$ is approaching or less than the resolution of the digital imaging microscope (Nikon 80i), which is restricted by the Abbe's formula. ${ }^{[3]}$ And limited to the fabrication errors in our experiment, the color appearance seems not uniform due to the lack of the size uniformity of the fabricated patterns. As mentioned above, the spectral filtering feature of the ultrathin metal patch array structure resulted from the localized surface plasmon 

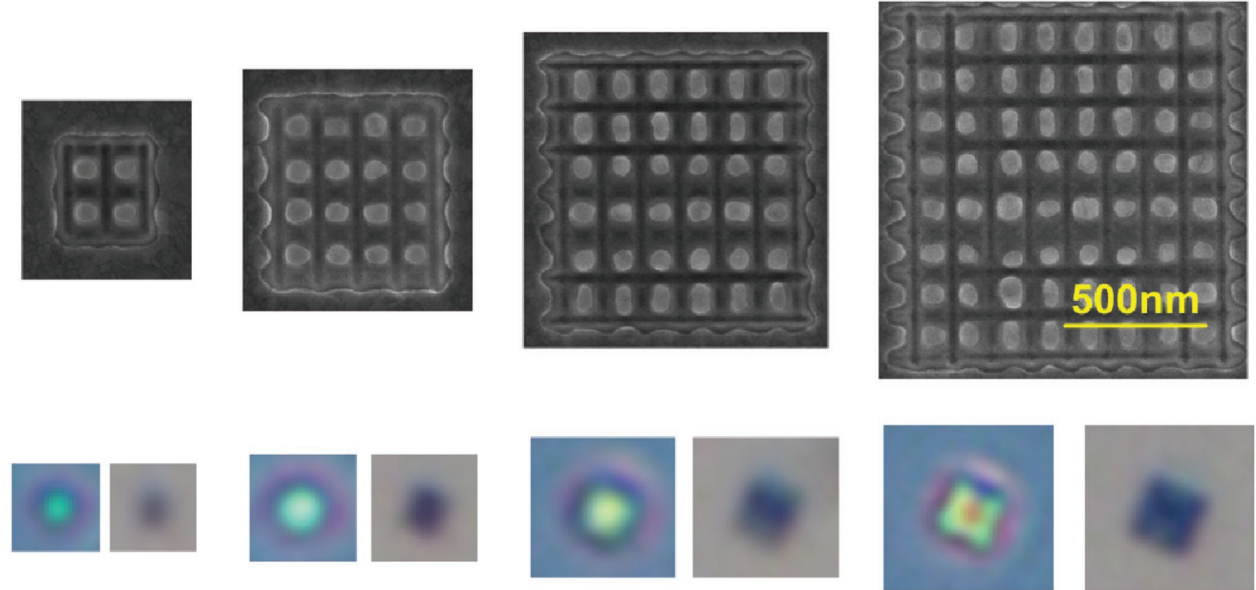

Figure 4. The SEM images with different pixel size and their corresponding photo images taken by digital imaging microscopy. The pixel size from left to right: $2 \times 2$ array, $4 \times 4$ array, $6 \times 6$ array, $8 \times 8$ array. The photo images of each group were taken in transmission and reflection respectively, from left to right.

resonance excited by single metallic patch. Therefore, the fact that the patch array with as few as two periods was sufficient to demonstrate color filtering is predictable. So, the great color pattern produced by the plasmonic structural color proposed in this paper can have a high resolution approaching the diffraction limit.

In conclusion, angle insensitive plasmonic filters using ultrathin metal patch array structure are proposed. The plasmonic filters can be used as reflective RGB filters as well as transmissive CMY filters simultaneously, and can present the same perceived reflection/transmission color at unpolarized illumination for a broad range of incident angles. The reflection/transmission curves are coincident at different angles and the color difference between different angels is pretty small. Various colors can be obtained by simply tuning the dimension of the patch structure. It is the localized surface plasmon resonance excited within the single metallic nanopatch that brings out the angle insensitive color filtering feature of this plasmonic filter. Moreover, patch arrays with as few as two periods are sufficient to demonstrate color filtering. As a matter of fact, nanoimprint technique as well as the column fabrication method based on the porous anodic alumina template and atomic layer deposition technique are expected for the preparation of this plasmonic filter with greatly reduced fabrication time and cost, taking the place of FIB milling technique. This method, described in this paper, can have tremendous potential for various applications in the fields of display, detecting, printing, decoration, and so forth.

\section{Experimental Section}

Simulation: Simulation of the reflectance and the angle resolved transmittance of the plasmonic filter was performed by FDTD method. In our simulation, the complex refractive index of the Ag material was obtained from the book published by CRC press. ${ }^{[25]}$ The electric field distribution profile of the structure was calculated by a commercial electromagnetic field calculation software, FDTD Solutions from Lumerical Inc.

Device Fabrication: The proposed angle robust plasmonic filter was manufactured on the clean substrates of fused silica. The ultrathin $\mathrm{Ag}$ film was deposited by magnetron sputtering with the base vacuum pressure better than $5 \times 10^{-4} \mathrm{~Pa}$. During the deposition, the substrates were kept at room temperature with the sputtering power set at $40 \mathrm{~W}$. After deposition, the pattern of the Ag film was processed by FIB milling using Carl Zeiss AURIGA Cross Beam (FIB-SEM) Workstation with the ion beam current $1.5 \mathrm{pA}$.

Optical Characterization: The reflectance measurement of the fabricated device at normal incidence was performed by the spectrophotometer (OLYMPUS USPM-RU), which was equipped with an optical microscopy. The angle resolved transmittance was measured by the self-made angle resolved measurement system.

\section{Supporting Information}

Supporting Information is available from the Wiley Online Library or from the author.

\section{Acknowledgements}

This work was supported by Zhejiang Provincial Natural Science Foundation (Grant No. LY13F050001), National High Technology Research and Development Program 863 (Grant No. 2015AA015904), and National Natural Science Foundation of China (Grant No. 61275161). The authors also acknowledge Mrs. Wei Wang for focused ion beam fabrication of the patch pattern and Dr. Bin Shi, Mr. Xiaolin Hu for angle resolved transmittance measurements.

Received: May 24, 2016

Revised: August 9, 2016

Published online: September 16, 2016

[1] K. T. Lee, S. Seo, J. Y. Lee, L. J. Guo, Adv. Mater. 2014, 26, 6324.

[2] K. Mao, W. Shen, C. Yang, X. Fang, W. Yuan, Y. Zhang, X. Liu, Sci. Rep. 2016, 6, 19289.

[3] C. Yang, W. Shen, Y. Zhang, K. Li, X. Fang, X. Zhang, X. Liu, Sci. Rep. 2015, 5, 9285 .

[4] W. L. Barnes, A. Dereux, T. W. Ebbesen, Nature 2003, 424, 824.

[5] K. Kumar, H. Duan, R. S. Hegde, S. C. Koh, J. N. Wei, J. K. Yang, Nat. Nanotechnol. 2012, 7, 557.

[6] T. Xu, Y.-K. Wu, X. Luo, L. J. Guo, Nat. Commun. 2010, 1, 59. 
[7] Y.-T. Yoon, C.-H. Park, S.-S. Lee, Appl. Phys. Express 2012, 5, 022501.

[8] J. Clausen, E. Højlund-Nielsen, A. Christiansen, S. Yazdi, M. Grajower, H. Taha, U. Levy, A. Kristensen, N. Mortensen, Nano Lett. 2014, 14, 4499.

[9] A. S. Roberts, A. Pors, O. Albrektsen, S. I. Bozhevolnyi, Nano Lett. 2014, 14, 783.

[10] J. Olson, A. Manjavacas, L. Liu, W.-S. Chang, B. Foerster, N. S. King, M. W. Knight, P. Nordlander, N. J. Halas, S. Link, Proc. Natl. Acad. Sci. 2014, 111, 14348.

[11] B. Zeng, Y. Gao, F. J. Bartoli, Sci. Rep. 2013, 3, 2840.

[12] V. R. Shrestha, S. Lee, E. Kim, D. Choi, Nano Lett. 2014, 14, 6672.

[13] J. Sauvage-Vincent, S. Tonchev, C. Veillas, S. Reynaud, Y. Jourlin, J. Eur. Opt. Soc.-Rapid 2013, 8, 13015.

[14] L. Duempelmann, D. Casari, A. Luu-Dinh, B. Gallinet, L. Novotny, ACS Nano 2015, 9, 12383

[15] Y. Ye, Y. Zhou, L. Chen, Appl. Opt. 2009, 48, 5035.

[16] T. Ellenbogen, K. Seo, K. B. Crozier, Nano Lett. 2012, 12, 1026.

[17] G. Y. Si, E. S. P. Leong, A. J. Danner, J. H. Teng, Proc. SPIE 2010, 7757, 77573F.

[18] Y. Chen, W. Liu, Opt. Lett. 2012, 37, 4.

[19] Q. Chen, D. R. S. Cumming, Opt. Express 2010, 18, 14056.

[20] L. Chen, Z. Qiang, H. Yang, H. Pang, Z. Ma, W. Zhou, Opt. Express 2009, 17, 8396.

[21] Y. Wu, A. Hollowell, C. Zhang, L J. Guo, Sci. Rep. 2013, 3, 1194.

[22] L. Duempelmann, A. Luu-Dinh, B. Gallinet, L. Novotny, ACS Photonics 2016, 3, 190.
[23] C. Yang, W. Shen, Y. Zhang, H. Peng, X. Zhang, X. Liu, Opt. Express 2014, 22, 11384.

[24] T. Cui, M. Qi, X. Wan, J. Zhao, Q. Cheng, Light: Sci. \& Appl. 2014, 3, e218.

[25] W. M. Haynes, CRC Handbook of Chemistry and Physics, CRC, Boca Raton, FL, USA 2014.

[26] C. Yang, L. Hong, W. Shen, Y. Zhang, X. Liu, H. Zhen, Opt. Express 2013, 21, 9315

[27] C. Yang, W. Shen, Y. Zhang, D. Zhao, X. Liu, Opt. Commun. 2014, 331, 310.

[28] A. Taflove, S. C. Hagness, Computational Electrodynamics: The Finite-Difference Time-Domain Method, Artech House, Norwood, MA, USA 2000.

[29] K. S. Kunz, R. J. Luebbers, The Finite Difference Time Domain Method for Electromagnetics, CRC, Boca Raton, FL, USA 1993.

[30] Y. Kane, IEEE Trans. Antennas and Propag. 1966, 14, 302.

[31] G. Sharma, W. Wu, E. N. Dalal, Color Res. Appl. 2005, 30, 21.

[32] M. R. Luo, G. Cui, B. Rigg, Color Res. Appl. 2001, 26, 340.

[33] CIE, Improvement to Industrial Colour Difference Evaluation, CIE, 2001.

[34] G. D’Aguanno, N. Mattiucci, A. Alù, M. J. Bloemer, Phys. Rev. B 2011, 83, 035426.

[35] J. Braun, B. Gompf, G. Kobiela, M. Dressel, Phys. Rev. Lett. 2009 103, 203901.

[36] M. Born, E. Wolf, Principles of Optics: Electromagnetic Theory of Propagation, Interference and Diffraction of Light, Cambridge University Press, Cambridge, UK 1999 